\title{
Biologia floral e polinização de Casearia grandiflora, Casearia. javitensis e Lindackeria paludosa (Flacourtiaceae) na região de Manaus, AM ${ }^{1}$
}

\author{
SILVIA PATRICIA FLORES VÁSQUEZ ${ }^{1,2,4}$ e ANTONIO CARLOS WEBBER ${ }^{3}$
}

(recebido: 04 de setembro de 2008; aceito: 11 de fevereiro de 2010)

\begin{abstract}
Floral biology and pollination of Casearia grandiflora, Casearia javitensis and Lindackeria paludosa (Flacourtiaceae) in the region of Manaus, AM). Floral biology, pollination and reproductive system of Casearia grandiflora Cambess., C. javitensis Kunth and Lindackeria paludosa (Benth.) Gilg were studied on the Federal University of Amazonas campus, Manaus,Amazonas, Brazil (0304'34" S, and 59 57'50” W), from November 2003 to December 2004. Observations of morphology, floral biology and floral visitors were made. The reproductive system and reproductive success was determined through field experimental pollinations. The flowers are hermaphrodites or staminated in Lindackeria paludosa and hermaphrodites in Casearia species, which emit a sweet scent during anthesis and has pollen of high fertility. Lindackeria paludosa and Casearia javitensis flowers open in the morning, whereas Casearia grandiflora flowers open at night. The main pollinators are flies of the Syrphidae family for the Casearia and bees of the Apidae and Halictidae families for L. paludosa. The results of the manual pollinations indicate that Casearia grandiflora and Lindackeria paludosa are auto-compatible species, in contrast to Caseria javitensis which is auto-incompatible. The species present a high reproductive potencial, but a low pre-emergent reproductive success.
\end{abstract}

Key words - reproductive success, reproductive sytem

RESUMO - (Biologia floral e polinização de Casearia grandiflora, Casearia javitensis e Lindackeria paludosa (Flacourtiaceae) na região de Manaus, AM). A biologia floral, polinização e sistema reprodutivo de Casearia grandiflora Cambess., C.javitensis Kunth e Lindackeria paludosa (Benth.) Gilg foram estudados no campus da Universidade Federal do Amazonas, Manaus, Brasil $\left(03^{\circ} 04^{\prime} 34^{\prime \prime} \mathrm{S}\right.$ e $\left.59^{\circ} 57^{\prime} 50^{\prime \prime} \mathrm{W}\right)$, durante o período de novembro 2003 a dezembro 2004 . Foram realizadas observações sobre morfologia, biologia floral e visitantes florais. O sistema reprodutivo e o sucesso reprodutivo foram determinados através de polinizações experimentais no campo. As flores são hermafroditas ou estaminadas em Lindackeria paludosa e hermafroditas nas espécies de Casearia que emitem um odor adocicado durante a antese e cujo pólen possui alta fertilidade. As flores de Lindackeria paludosa e Casearia javitensis abrem durante a manhã, enquanto que as de Casearia grandiflora abrem durante a noite. Os principais polinizadores são moscas da família Syrphidae em espécies de Casearia e abelhas das famílias Apidae e Halictidae em L. paludosa. Os resultados das polinizações manuais indicam que Casearia grandiflora e Lindackeria paludosa são espécies autocompatíveis, ao contrário de Casearia javitensis, que é autoincompatível. As espécies apresentaram um alto potencial reprodutivo, mas baixo sucesso reprodutivo pré-emergente.

Palavras-chave - sistema reprodutivo, sucesso reprodutivo

\section{Introdução}

As florestas tropicais constituem um dos ecossistemas mais ricos em biodiversidade. Nos últimos anos a sua exploração e destruição têm se tornado um dos maiores problemas mundiais (Delamonica et al. 2001). Particularmente, o ecossistema Amazônico tem despertado maior interesse porque abriga extraordinária

1. Parte da dissertação do primeiro autor, Programa de Pós-Graduação em Biologia Tropical e Recursos Naturais na área de Botânica, do Instituto Nacional de Pesquisas da Amazônia, Manaus, AM, Brasil.

2. Instituto Nacional de Pesquisas da Amazônia, INPA, Av. André Araújo, 2936, 69060-000 Manaus, AM, Brasil.

3. Universidade Federal do Amazonas, Departamento de Biologia, ICB, Av. Gal. Rodrigo Jordão Ramos, 3000, 69077-000 Manaus, AM, Brasil.

4. Autor para correspondência: silviapfv@yahoo.com.br diversidade (Salati et al. 1998). O estudo da biologia reprodutiva de árvores tropicais tem sido objeto de grande interesse na comunidade científica para atender a crescente necessidade de compreender como é possível manter a biodiversidade (Bawa 1990). Os dados sobre a reprodução de uma espécie, como sistema sexual, mecanismo de incompatibilidade, padrão de floração e processo de polinização podem nortear a adoção de estratégias de manejo e manutenção de populações e ecossistemas (Hamrick \& Nason 2000).

A diversidade de espécies da floresta tropical é acompanhada por uma grande diversidade de mecanismos de polinização e é surpreendente que exista uma carência de dados acerca da polinização de muitos grupos importantes de plantas (Prance 1985). Sistemas de polinização envolvem desde insetos pouco especialistas até animais estritamente dependente das flores para sua 
sobrevivência, como abelhas e mesmo vertebrados, como aves e morcegos (Pires-O'Brien \& O'Brien 1995). Portanto, a polinização é uma componente chave da biodiversidade, sendo diretamente responsável pela manutenção dos ecossistemas (Bawa et al. 1990).

Existem poucos estudos sobre polinização e sistema reprodutivo de representantes de Flacourtiaceae (sensu Sleumer 1980). Na Amazônia colombiana, Homalium guianense (Aub.) Oken é espécie hermafrodita e polinizada por diversos insetos pequenos (Dulmen 2001); na Malásia, Casearia grewiifolia Vent. e Hydnocarpus borneensis Sleumer são polinizadas por abelhas sociais e diversos outros insetos (Momose et al. 1998); no México, em uma floresta tropical decídua foram registradas várias espécies de Casearia como hermafroditas e autoincompatíveis (Bullock 1985); no Parque Nacional Sarawak, Casearia grewiifolia é um subarbusto com flor actinomorfa, hermafrodita e possui pouco néctar (Kato 1996); e em uma floresta secundária decídua na Venezuela, Prockia flava H. Karst. é reportada como hermafrodita, autoincompatível e polinizada por abelhas da família Apidae (Zapata \& Arroyo 1978). No Brasil, Machado \& Oliveira (2000) estudaram a biologia floral e reprodutiva de Casearia grandiflora Cambess., num remanescente de mata mesófila em Minas Gerais

Este trabalho tem como objetivo descrever alguns aspectos da biologia floral, polinização e sistema reprodutivo de Casearia grandiflora, $C$.javitensis Kunth e Lindackeria paludosa (Benth.) Gilg.

\section{Material e métodos}

O trabalho de campo foi realizado no campus da Universidade Federal do Amazonas - UFAM ( $\left(03^{\circ} 04^{\prime} 34^{\prime \prime}\right.$ S e $\left.59^{\circ} 57^{\prime} 50^{\prime \prime} \mathrm{W}\right)$, no Município de Manaus, AM. A vegetação do campus da UFAM é constituída por floresta de platô, floresta aberta, campinarana, áreas de vegetação secundária e área antrópica (Silva-Forsberg 1999). As espécies estudadas ocorrem em vegetação secundária. Manaus tem clima do tipo Am, quente e úmido ao longo de todo o ano (classificação de Köppen). A temperatura e a precipitação média anual são de $26{ }^{\circ} \mathrm{C}$ e $2100 \mathrm{~mm}$, respectivamente (Silva-Forsberg 1999). As observações de campo foram semanais, no período de novembro de 2003 a dezembro de 2004, e estenderam-se das 06h00 às 18h00, incluindo observações noturnas de $22 \mathrm{~h} 00$ às 5h00. Material testemunho de Casearia grandiflora, $C$. javitensis e Lindackeria paludosa foi depositado no herbário do Instituto Nacional de Pesquisas da Amazônia (INPA 215222, 215223, 215224).

Para a descrição morfológica, 10 flores de seis indivíduos de cada espécie foram armazenadas em etanol $70 \%$. Informações sobre período de disponibilidade de néctar e liberação de pólen foram registradas através de observações diretas no campo em 10 flores de cinco indivíduos de cada espécie. O horário e o processo da abertura floral e a duração da flor foram definidos após a marcação de 10 botões em três indivíduos de cada espécie. A receptividade do estigma foi testada, ao longo do dia com peróxido de hidrogênio (Dafni 1992). Para tanto foram utilizadas 10 flores de seis indivíduos de cada espécie.

Para o registro do volume de néctar foram ensacadas 165 flores em pré-antese de dois indivíduos de C.javitensis. As primeiras medidas foram efetuadas no início da antese, por volta das $07 \mathrm{~h} 00$, sendo estas repetidas a cada hora, finalizando às $18 \mathrm{~h} 00$. O néctar foi totalmente retirado com auxílio de microcapilares de 1 e $2 \mu \mathrm{L}$. Para $C$. grandiflora o néctar foi coletado uma única vez em 30 flores previamente ensacadas de um indivíduo, devido à pouca quantidade de néctar por flor. A porcentagem de açúcares dissolvidos foi medida em 30 flores de três indivíduos de cada espécie de Casearia com auxílio de refratômetro manual Digit modelo 107 (Kearns \& Inouye 1993). Em L. paludosa não foi realizado este teste, pois as flores só apresentam pólen como recompensa. A presença de osmóforos foi detectada com a utilização do vermelho neutro (Dafni 1992), em 10 flores de cinco indivíduos de cada espécie.

Para a análise da fertilidade dos grãos de pólen, as anteras de três flores de cada espécie foram colocadas em lâminas, coradas com azul de algodão-lactofenol e permaneceram por um dia para verificação da coloração. Este método considera como potencialmente férteis os grãos corados de azul intenso e não viáveis os incolores (Kearns \& Inouye 1993). A porcentagem de pólen viável foi obtida a partir de um lote de 250 grãos por lâmina).

Observações do comportamento dos visitantes foram feitas em cinco indivíduos de cada espécie durante todo o período de floração, desde o início da antese até a senescência das flores, entre $05 \mathrm{~h} 00$ e $18 \mathrm{~h} 00$, totalizando 130 horas de observação. Os dados sobre o tipo de recurso procurado, o horário e o número de visitas e o tempo de permanência nas flores foram obtidos por observações diretas e complementados com análise de fotografias. Cada pouso do visitante na flor foi considerado como uma visita, sendo possível registrar número de visitas por intervalo de tempo. Foi verificada a presença de pólen no corpo dos insetos, o qual foi comparado com o pólen das espécies estudadas. O pólen transportado pelos insetos foi coletado com auxílio de pequenos cubos de gelatina (Kears \& Inouye 1993). Os insetos visitantes foram capturados com auxílio de rede entomológica, sacos e frascos coletores, posteriormente identificados por especialistas e depositados na coleção entomológica do INPA.

Para o estudo do sistema reprodutivo foram utilizadas flores de cinco indivíduos de $C$. grandiflora, três de $L$. paludosa e dois de C. javitensis. Em cada tratamento foram isolados entre 150 a 200 botões, um ou dois dias antes da antese, utilizando-se sacos de organza. Não foi possível realizar todos os testes de polinizações manuais devido aos danos causados aos experimentos por pessoas na área. As 
flores foram utilizadas para os seguintes tratamentos: (a) autopolinização espontânea: as flores foram ensacadas para verificar a formação de frutos; (b) autopolinização manual: depositando pólen de uma flor sobre seu próprio estigma; (c) controle: as flores foram etiquetadas para verificar a eficácia dos polinizadores. Para os tratamentos de polinização com L. paludosa foram utilizadas apenas flores hermafroditas, e no caso de autopolinização espontânea, as flores não foram emasculadas devido ao seu tamanho reduzido. As diferenças na proporção de frutos formados entre os tratamentos (autopolinizações manuais e controle) foram determinadas através de Análise de Variância (ANOVA) a 5\% e pelo teste de Tuckey, utilizando o programa estatístico Prophet versão 5.

Para quantificar o potencial e o sucesso reprodutivo em condições naturais foram marcados dez indivíduos de C. grandiflora, oito de L. paludosa e cinco de C. javitensis. $\mathrm{O}$ número de indivíduos marcados dependeu do número de indivíduos floridos. Foram selecionados cinco ramos em cada indivíduo. Cinco inflorescências na fase de botão desenvolvido em cada ramo foram etiquetadas com um fio colorido. $\mathrm{O}$ número de botões florais por inflorescência foi contado. Após três dias da abertura da flor o número de pistilos que permaneceram foram contados e, posteriormente, foram contados os números de frutos e de sementes por fruto. O sucesso reprodutivo pré-emergente (número de sementes viáveis que entram no meio ambiente) foi calculado pela razão de flores por frutos $(\mathrm{Fl} / \mathrm{Fr})$ multiplicados pela razão de sementes por óvulos (S/O) (Wiens et al. 1987). Não foi feito teste de viabilidade de sementes e foram consideradas viáveis aquelas que não apresentavam danos externos aparentes.

\section{Resultados e discussão}

Morfologia floral - As inflorescências são axilares do tipo racemo em L. paludosa e glomeruladas em C. grandiflora e C. javitensis. O número de botões por inflorescência, diâmetro das flores e as medidas das peças florais encontram-se na tabela 1. As flores de C. grandiflora e C. javitensis são hermafroditas, monoclamídeas, amareladas ou esverdeadas, com sépalas unidas na base formando um tubo curto em $C$. grandiflora e livres em C.javitensis. Essas características também foram descritas por Sleumer (1980), entretanto divergem do resultado apresentado por Machado \& Oliveira (2000). Esses autores descrevem erroneamente C. grandiflora como diclamídea, com corola pentâmera e pétalas unidas na base. As flores em L. paludosa são hermafroditas ou estaminadas, diclamídeas, dialissépalas e dialipétalas, brancas. Nas flores estaminadas o androceu é composto por numerosos estames livres, com pistilódio rudimentar.

Nas flores hermafroditas de todas as espécies o androceu é dialistêmone, com os estames dispostos ao redor do gineceu e as anteras possuem deiscência longitudinal. O gineceu apresenta ovário súpero, tricarpelar, com placentação parietal. O fruto é uma cápsula ovóide, trivalvada. As flores de C. grandiflora e $C$. javitensis apresentam atributos que as enquadram na síndrome de Miofilia descrita por Faegri \& van der Pijl (1979). Não foi possível enquadrar as flores de L. paludosa em nenhuma síndrome.

Biologia floral - A antese das espécies de Casearia começa com a separação das sépalas, que lentamente abrem e expõem o estigma e os estames. As flores de C. grandiflora abrem durante a noite, a receptividade do estigma começa às $03 \mathrm{~h} 00$, de cor amarelo e com a superfície coberta por secreção brilhante e úmida, as anteras abrem às $04 \mathrm{~h} 00$ e o pólen está completamente liberado em torno das 09 h00 e 10 h00. A duração da flor foi de $18 \mathrm{~h}$. Estes dados diferem dos encontrados por (Machado \& Oliveira 2000) para a mesma espécie. Esses autores reportaram que a antese ocorreu de forma irregular, principalmente no início da manhã e

Tabela 1. Número de botões por inflorescência, e medidas ( $\bar{x} \pm \mathrm{DP})$ dos verticilos florais em 10 flores de Lindackeria paludosa, Casearia grandiflora e C. javitensis no campus da UFAM, Manaus, AM.

Table 1. Number of buds in inflorescences, and measures $(\bar{x} \pm \mathrm{DP})$ of perianth parts in 10 flowers of Lindackeria paludosa, Casearia grandiflora and $C$. javitensis at the UFAM campus, Manaus, AM.

\begin{tabular}{lccc}
\hline Caracteres & L. paludosa & C. grandiflora & C. javitensis \\
\hline Botões/infl. $(n=10)$ & $7-40(21 \pm 9,6)$ & $1-18(9,7 \pm 4,4)$ & $6-40(21 \pm 12,9)$ \\
Diâmetro floral $(\mathrm{mm})$ & $11,7( \pm 1,2)$ & $6,9( \pm 0,9)$ & $5,3( \pm 0,9)$ \\
Sépala & $5,4( \pm 0,9)$ & $6,1( \pm 0,3)$ & $3,3( \pm 0,4)$ \\
Pétala & $5,9( \pm 0,7)$ & - & - \\
Estame & $4,7( \pm 0,8)$ & $3,6( \pm 0,5)$ & $5,9( \pm 0,5)$ \\
Pistilo & $6,8( \pm 0,6)$ & $5,2( \pm 0,4)$ & $4,9( \pm 0,3)$ \\
\hline
\end{tabular}


que a receptividade do estigma e a liberação do pólen ocorreram nas horas mais quentes do dia. Em L.paludosa, a antese iniciou as $09 \mathrm{~h} 00$, ficando a flor completamente aberta às $05 \mathrm{~h} 30$ do dia seguinte com duração de um dia e meio, a receptividade do estigma ocorreu nos botões em pré-antese, sendo a liberação do pólen entre $06 \mathrm{~h} 00 \mathrm{e}$ 07h00. Em C.javitensis as flores iniciaram a abertura por volta das $09 \mathrm{~h} 00$, estando a flor completamente aberta às 04h00 do dia seguinte com duração de um dia e meio, a receptividade do estigma e a liberação completa do pólen ocorreram às 05h00. Martiniano (2004) descreveu que as flores de Ryania speciosa Vahl (Flacourtiaceae) abrem às 05 h00 com duração de um dia; as anteras abrem entre 08h00 e 09h00.Durante o período de antese, os grãos de pólen das espécies aqui estudadas estão totalmente expostos, não havendo, assim, quaisquer restrições à sua coleta, característica esta comum das flores generalistas (Faegri \& van der Pij1 1979, Endress 1994). As espécies apresentam fertilidade do pólen superior a $90 \%$.

Nas primeiras horas da manhã as flores exalam um odor agradável e perfumado. Os osmóforos foram localizados nas sépalas em C. grandiflora e C.javitensis e nas sépalas, pétalas e estames em L. paludosa. Segundo Machado \& Oliveira (2000), o odor das flores de C. grandiflora foi imperceptível ao observador.

Em C. grandiflora o néctar é produzido na base das sépalas que começam a secretá-lo nas primeiras horas da manhã em pequenas quantidades. O volume foi de $4 \mu \mathrm{L}$, a concentração de açúcar foi de $54 \%$. Uma medição pela manhã (08h00) indicou 40\% de açúcares. Dados similares foram encontrados por Machado \& Oliveira (2000), para a mesma espécie e reportaram que o néctar é relativamente abundante $(4 \mu \mathrm{L} \pm 0,4)$ e com concentração média de $38 \%$. Em C. javitensis a produção de néctar pode ser detectada a partir do início da abertura da flor. A concentração de açúcar foi em torno de $51 \%$. O volume total de néctar produzido durante a antese foi de $28,5 \mu \mathrm{L}$ que de 2,6 $\pm 1,5$ foi aumentando ao longo da manhã (figura 1). Estes dados diferem dos encontrados para Ryania speciosa (Martiniano 2004) onde a porcentagem permanece inalterada até o meio dia e logo começa a diminuir. $\mathrm{O}$ aumento na concentração de açúcar no néctar pode estar relacionado a mudanças de temperatura (Baker \& Baker 1983) e umidade relativa (Corbet et al. 1979). O néctar é considerado atrativo primário por servir como alimento para muitos animais que visitam as flores repetidamente (Faegri \& van der Pij1 1979). O volume de néctar por flor produzido por C. grandiflora e C.javitensis é baixo, mas é compensado pela alta concentração de açúcares. A baixa quantidade de néctar por flor é atributo que pode ser importante

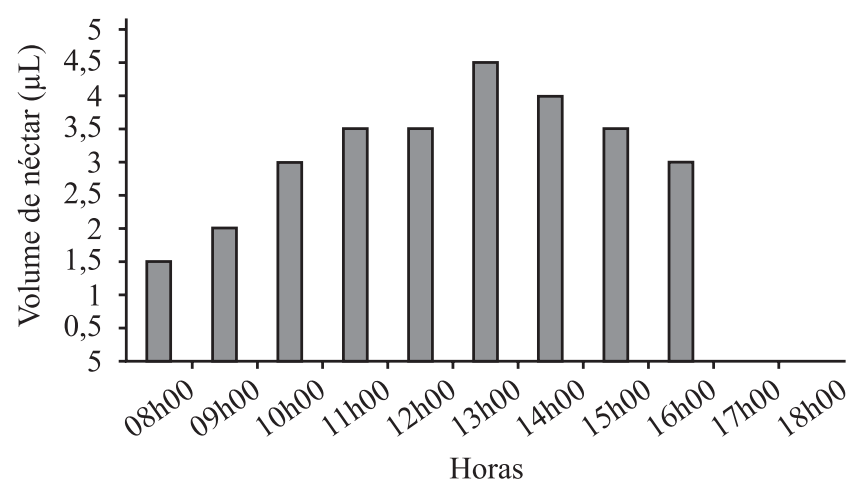

Figura 1. Produção de néctar ao longo do dia em 165 flores de Casearia javitensis no campus da UFAM, Manaus, AM.

Figure 1. Nectar production during a day in 165 flowers of Casearia javitensis at the campus of the UFAM, Manaus, AM.

na seleção dos polinizadores (Sigrist \& Sazima 2002), e pode representar uma estratégia para que os animais visitem flores de outros indivíduos em busca de mais recursos. Com isso, gera um grande movimento entre os indivíduos da mesma espécie e promove a polinização cruzada (Santos \& Machado 1998).

O processo de senescência das flores ocorre no final da tarde, quando ocorrem mudanças de coloração dos estames, sépalas e pétalas para marrom escuro. Os estiletes permanecem até a formação do fruto em todas as espécies estudadas.

Insetos visitantes - Diversos grupos de insetos foram observados visitando as flores de C. grandiflora e C. javitensis, sendo o principal polinizador a mosca Ornidia obesa (Syrphidae) (tabela 2). O horário com maior número de visitas em $C$. grandiflora ocorreu entre $10 \mathrm{~h} 00$ e $12 \mathrm{~h} 00$ (figura 2). O principal recurso coletado pelos visitantes foi o néctar, mais também foi coletado pólen.

Para realizar a coleta, a mosca $O$. obesa chegava à planta por volta das $05 \mathrm{~h} 30$, pousava nas sépalas e com as pernas dianteiras e a probóscide pegava o pólen estame por estame ao mesmo tempo que introduzia a probóscide na flor para a coleta de néctar. Durante esse procedimento, contatava o estigma e as anteras. Após ficar de dois a três minutos na flor, voava para outra percorrendo toda a inflorescência com o mesmo comportamento. De vez em quando, voava, pousava na parte adaxial de uma folha e limpava a probóscide, cabeça e pernas. Machado \& Oliveira (2000) também reportaram que o visitante mais freqüente foi a mosca $O$. obesa e que estas foram observadas repetidamente 
Tabela 2. Visitantes e possíveis polinizadores de Lindackeria paludosa, Casearia grandiflora e C. javitensis no campus da UFAM, Manaus, AM.

Table 2. Visitors and possible pollinators of Lindackeria paludosa, Casearia grandiflora and C. javitensis at the UFAM campus, Manaus, AM.

\begin{tabular}{|c|c|c|c|}
\hline Visitantes & L. paludosa & C. grandiflora & C. javitensis \\
\hline \multicolumn{4}{|l|}{ COLEOPTERA } \\
\hline \multicolumn{4}{|l|}{ Brentidae } \\
\hline Morfotipo 1 & & $\mathrm{x}$ & \\
\hline Morfotipo 2 & & $\mathrm{x}$ & \\
\hline Cerambycidae & & & $\mathrm{x}$ \\
\hline Curculionidae & & & $\mathrm{x}$ \\
\hline Mionochroma vittatum Fabricius 1775 & & & $\mathrm{x}$ \\
\hline \multicolumn{4}{|l|}{ DIPTERA } \\
\hline \multicolumn{4}{|l|}{ Calliphoridae } \\
\hline Chrysomya megacephala Fabricius 1794 & & & $\mathrm{x}$ \\
\hline Paralucilia sp. & & & $\mathrm{x}$ \\
\hline \multicolumn{4}{|l|}{ Stratiomydae } \\
\hline Hoplitimyia subalba (Walker, 1854) & & & $\mathrm{x}$ \\
\hline \multicolumn{4}{|l|}{ Syrphidae } \\
\hline Ornidia obesa Lepeletier \& Serville, 1828 & & $\mathrm{x}$ & $\mathrm{x}$ \\
\hline Palpada vinetorum Frabricius, 1798 & & $\mathrm{x}$ & $\mathrm{x}$ \\
\hline \multicolumn{4}{|l|}{ HYMENOPTERA } \\
\hline \multicolumn{4}{|l|}{ Apidae } \\
\hline Apis mellifera scutellata Lepeletier, 1836 & & $\mathrm{x}$ & $\mathrm{x}$ \\
\hline Cephalotrigona capitata femorata (Smith, 1854) & & $\mathrm{x}$ & \\
\hline Exomalopsis sp. & $\mathrm{x}$ & & \\
\hline Friseomelitta sp. & $\mathrm{x}$ & & \\
\hline Melipona compressipes manaosensis Schwarz, 1932 & $\mathrm{x}$ & & \\
\hline Melipona puncticollis Friese, 1902 & & & $\mathrm{x}$ \\
\hline Melipona seminigra merrillae Cockerell, 1919 & $\mathrm{x}$ & $\mathrm{x}$ & $\mathrm{x}$ \\
\hline Partamona vicina Camargo, 1980 & & & $\mathrm{x}$ \\
\hline Ptilitrigona lurida (Smith, 1854) & & & $\mathrm{x}$ \\
\hline Trigona amazonensis Ducke, 1916 & & $\mathrm{x}$ & \\
\hline Trigona fulviventris Guerin, 1835 & $\mathrm{x}$ & & $\mathrm{x}$ \\
\hline Scaptotrigona sp. & $\mathrm{x}$ & & $\mathrm{x}$ \\
\hline Xylocopa sp. 1 & $\mathrm{x}$ & & \\
\hline Xylocopa sp. 2 & & $\mathrm{x}$ & $\mathrm{x}$ \\
\hline \multicolumn{4}{|l|}{ Halictidae } \\
\hline Augochloropsis sp. & $\mathrm{x}$ & & \\
\hline Stilbochlora sp. & $\mathrm{x}$ & & \\
\hline \multicolumn{4}{|l|}{ Vespidae } \\
\hline Morfotipo 1 & & $\mathrm{x}$ & $\mathrm{x}$ \\
\hline Morfotipo 2 & & $\mathrm{x}$ & \\
\hline Morfotipo 3 & & $\mathrm{x}$ & \\
\hline \multicolumn{4}{|l|}{ Sphecidae } \\
\hline Dynatus nigripes nigripes (Westwood, 1832) & & $\mathrm{x}$ & \\
\hline \multicolumn{4}{|l|}{ LEPIDOPTERA } \\
\hline \multicolumn{4}{|l|}{ Arctiidae } \\
\hline Morfotipo 1 & & $\mathrm{x}$ & $\mathrm{x}$ \\
\hline \multicolumn{4}{|l|}{ Hesperiidae } \\
\hline Morfotipo 1 & & & $\mathrm{x}$ \\
\hline \multicolumn{4}{|l|}{ Lycaenidae } \\
\hline Morfotipo 1 & & & $\mathrm{x}$ \\
\hline
\end{tabular}




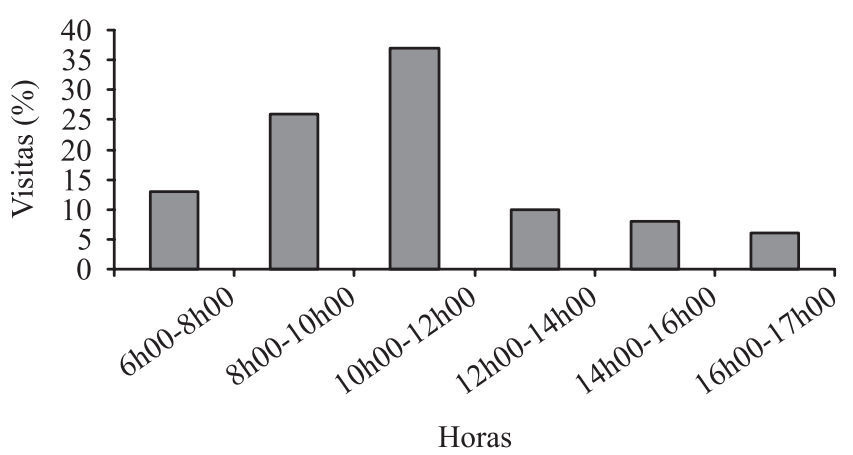

Figura 2. Porcentagem de visitas às flores de Casearia grandiflora por intervalo de tempo no campus da UFAM, Manaus, AM.

Figure 2. Percentage of visits to the flowers of Casearia grandiflora for time interval at the campus of the UFAM, Manaus, AM.

em todas as plantas acompanhadas durante o estudo e visitavam as flores durante quase todo o dia. A ocorrência de polinização por moscas em espécies arbóreas de flores aparentemente generalistas tem sido observada para outras espécies com flores pequenas (Pombal 1994, Pombal \& Morellato 1995). Entre elas há o levantamento feito na Estação Ecológica da Universidade Federal de Minas Gerais em Belo Horizonte por Silva et al. (2001) que registraram 40 espécies de moscas visitando 16 famílias de plantas, sendo registradas com maior freqüência O. obesa e Palpada furcata (ambas da família Syrphidae). Machado \& Loiola (2000), no estudo feito com Cordia multispicata Cham. (Boraginaceae) e Borreria alata (Aubl.) DC. (Rubiaceae), citaram nove espécies de moscas, principalmente do gênero Palpada. Muitas espécies da família Syrphidae visitam variadas espécies de plantas nas quais o acesso ao néctar e ao pólen é mais fácil (Silva et al. 2001), como é o caso das flores de $C$. grandiflora e $C$.javitensis. Além das moscas também foram observados besouros, vespas e borboletas (figuras 3 e 4) visitando as flores de $C$. grandiflora e C. javitensis, sendo consideradas como polinizadores ocasionais por suas visitas menos freqüentes. Dentre as abelhas, Melipona seminigra merrillae (figura 5) e Cephalotrigona capitata femorata (figura 6) foram as mais freqüentes, sendo considerados polinizadores. Foi encontrado pólen das espécies de Casearia no aparelho bucal, cabeça e pernas dos visitantes.

Visitas de diferentes grupos de insetos também foram citadas para Ryania speciosa (Martiniano 2004), Casearia corymbosa Kunth (Bawa et al. 1985b), C. grewiifolia e Hydnocarpus bornensis (Kato 1996,
Momose et al. 1998) e para Homalium guianense (Dulmen 2001); essas espécies são polinizadas por diversos insetos (pequenas abelhas, besouros, moscas, vespas e borboletas). Machado \& Oliveira (2000), no estudo feito em Uberlândia, também verificaram a presença de abelhas Meliponinae, borboletas e moscas não identificadas em flores de $C$. grandiflora.

Entre os visitantes de L. paludosa destacaram-se as abelhas das famílias Apidae e Halictidae, sendo $M$. seminigra merrillae o principal polinizador (tabela 2). As espécies de Melipona e Xylocopa sobrevoavam a planta e para a coleta de pólen pousavam nos estames, tocando as anteras com as mandíbulas e as pernas com ligeiros movimentos. Este movimento provocava uma nuvem rápida e esparsa de pólen (sem o evidente processo de vibração), ficando aderido em todo o corpo. O estigma era contatado várias vezes no momento da coleta do pólen, portanto são polinizadores. Após a coleta caminhavam pelo ramo ou voavam procurando outra flor. Augochloropsis sp. (figura 7) pousava sobre os estames e coletava o pólen de cada um com as mandíbulas e os dois primeiros pares de pernas, contatando o estigma. Trigona fulviventris (figura 8), Exomalopsis sp., Scaptotrigona sp. e Stilbochlora sp., foram os visitantes menos freqüentes (figura 9). A análise das lâminas de pólen coletada das abelhas mostrou que estava presente o pólen de L. paludosa. O recurso oferecido pelas flores aos seus visitantes é o pólen. Thorp (2000) relatou que os grãos de pólen são fontes ricas em nitrogênio/proteína, vitaminas, carboidratos e lipídios onde são utilizados, principalmente, como alimento para as larvas de abelhas, moscas, besouros, vespas, pássaros e morcegos. Vários estudos feitos com outras espécies de Flacourtiaceae também citam a presença de abelhas como polinizadores (Zapata \& Arroyo 1978, Bawa et al. 1985b, Kato 1996, Momose et al. 1998, Dulmen 2001).

Sistema reprodutivo - Os resultados dos experimentos de polinização manual das três espécies encontram-se na tabela 3. No controle da espécie $C$. grandiflora teve maior número de frutos formados com diferença significativa dos tratamentos de autopolinização manual e autopolinização espontânea. Nas demais espécies não foi verificada essa diferença. De acordo com estes resultados, C. grandiflora e L. paludosa são espécies autocompatíveis, diferindo dos resultados encontrados por Machado \& Oliveira (2000), que mostraram que C. grandiflora é autoincompatível. No entanto esses autores observaram tubos polínicos crescendo até o ovário e penetrando os óvulos em flores autopolinizadas, sugerindo a ocorrência de autoincompatíbilidade de ação 

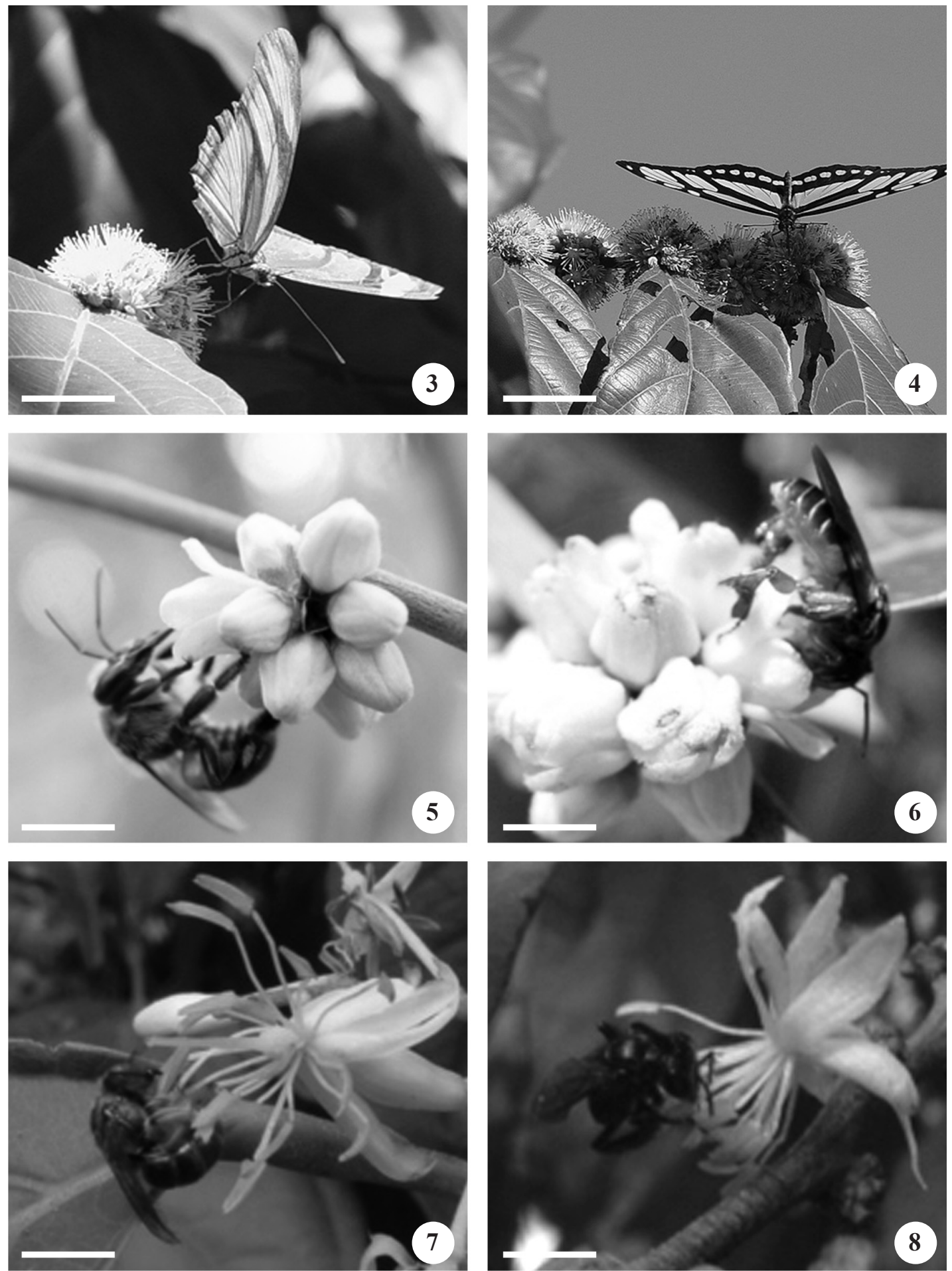

Figuras 3-8. 3. Borboleta Arctiidae visitando flores de Casearia javitensis. 4. Borboleta Lycaenidae coletando néctar de C. javitensis. 5. Melipona seminigra merrilae coletando néctar de Casearia grandiflora. 6. Cephalotrigona capitata femorata, visitando flores de C. grandiflora. 7. Augocloropsis sp. visitando flores de Lindackeria paludosa. 8. Trigona fulviventris, coletando pólen de L. paludosa. Barra =10 $\mathrm{mm}(3,4) ; 6 \mathrm{~mm}(5,6) ; 16 \mathrm{~mm}(7,8)$.

Figures 3-8. 3. Arctiidae butterfly visiting flowers from Casearia javitensis. 4. Lycaenidae butterfly collecting nectar from C. javitensis. 5. Melipona seminigra merrilae collecting nectar from Casearia grandiflora. 6. Cephalotrigona capitata femorata, visiting f lowers from C. grandiflora. 7. Augocloropsis sp. visiting flowers from Lindackeria paludosa. 8. Trigona fulviventris, collecting polen from $L$. paludosa. Bar $=10 \mathrm{~mm}(3,4) ; 6 \mathrm{~mm}(5,6) ; 16 \mathrm{~mm}(7,8)$. 


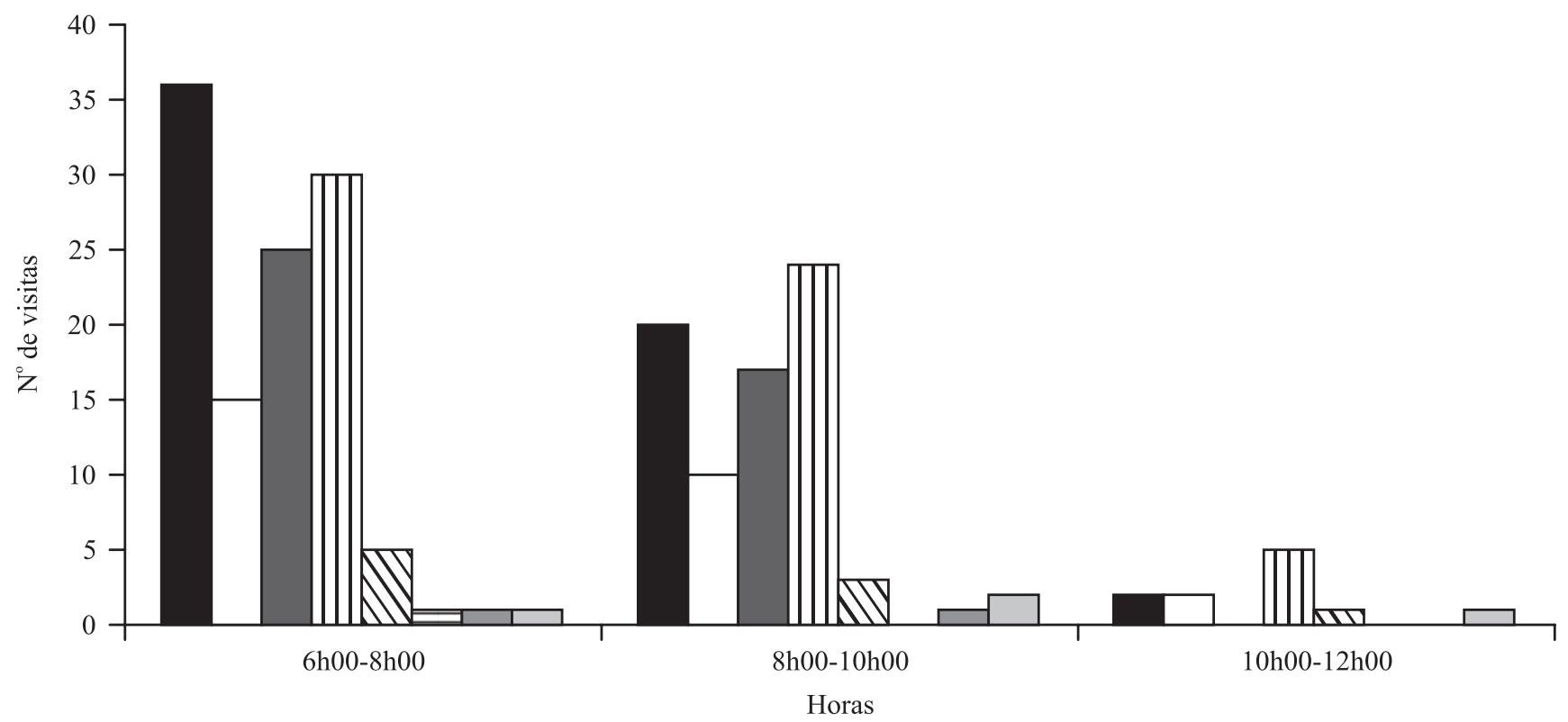

Figura 9. Número de visitas às flores de Lindackeria paludosa por intervalo de tempo no campus da UFAM, Manaus, AM. ( $\mathbf{\square}=$ Melipona seminigra merrillae; $\square=$ Melipona compressipes manaosensis; $\square=$ Xylocopa $\mathrm{sp} . ; \mathbf{m}=$ Augochloropsis sp.; $\mathbb{Q}=$ Trigona fulviventris; $\mathrm{\Xi}=$ Exomalopsis sp.; $\square=$ Scaptotrigona $; \square=$ Stilbochlora $\mathrm{sp}$. .).

Figure 9. Number of visits to the flowers of Lindackeria paludosa at time intervals at the campus of the UFAM, Manaus, AM. ( = Melipona seminigra merrillae; $\square=$ Melipona compressipes manaosensis; $\square$ Xylocopa sp.; 血 Augochloropsis sp.;

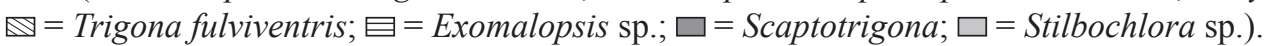

Tabela 3. Testes para avaliar o sistema reprodutivo de Lindackeria paludosa, Casearia grandiflora e C. javitensis no campus da UFAM, Manaus, AM.

Table 3. Tests to evaluate the reproductive system of Lindackeria paludosa, Casearia grandiflora and C. javitensis at the UFAM campus, Manaus, AM.

\begin{tabular}{|c|c|c|c|c|c|c|}
\hline \multirow[b]{2}{*}{ Testes } & \multicolumn{2}{|c|}{ L. paludosa } & \multicolumn{2}{|c|}{ C. grandiflora } & \multicolumn{2}{|c|}{ C. javitensis } \\
\hline & $\begin{array}{c}\text { Flores } \\
\mathrm{n}^{\mathbf{0}}\end{array}$ & $\begin{array}{l}\text { Frutos } \\
\text { no }(\%)\end{array}$ & $\begin{array}{c}\text { Flores } \\
\mathrm{n}^{\mathrm{o}}\end{array}$ & $\begin{array}{l}\text { Frutos } \\
\text { no }(\%)\end{array}$ & $\begin{array}{c}\text { Flores } \\
\mathrm{n}^{\mathrm{o}}\end{array}$ & $\begin{array}{l}\text { Frutos } \\
\mathrm{n}^{\circ}(\%)\end{array}$ \\
\hline Autopolinização manual & 508 & $105(21) \mathrm{ab}^{1}$ & 1066 & $22(2) b$ & 230 & $0 \mathrm{~b}$ \\
\hline Autopolinização espontânea & 801 & $198(25) \mathrm{ab}$ & 1055 & $3(0,2) b$ & 450 & $1(0,02) b$ \\
\hline Controle & 978 & 175 (18)ab & 11175 & 38 (48)a & 400 & $16(4) b$ \\
\hline
\end{tabular}

${ }^{1}$ Médias com letras distintas, na coluna, diferem entre si pelo teste de Tukey a $5 \%, P(0,02) .{ }^{1}$ Means with distinct letters in the columns are different according to Tukey test at $5 \%, P(0,02)$.

tardia ou de depressão endogâmica. Diferentes graus de compatibilidade podem ser encontrados entre as angiospermas e a distinção entre autoincompatibilidade e autocompatibilidade é muitas vezes arbitrária (Borba et al. 2001), pois esses são dois extremos na variação do sistema reprodutivo e muitas espécies se situam entre eles (Bawa 1974). Uma mesma espécie pode apresentar uma ou outra condição em diferentes regiões geográficas se, por exemplo, faltam os serviços dos polinizadores (Medrano et al. 1999). Segundo Jaimes \& Ramirez (1999), espécies autocompatíveis estão associadas a estágios de sucessão inicial ou áreas com distúrbio, onde autogamia é seletiva como estratégia de colonização. Esse pode ser o caso das duas espécies que são autocompatíveis com grande potencial de colonização de áreas. Entretanto, C. javitensis é autoincompatível, uma vez que nos 
testes de autopolinização não houve formação de frutos (tabela 3). Resultados semelhantes foram encontrados para C. grandiflora (Machado \& Oliveira 2000), C. corymbosa, C. tremula (Griseb.) Griseb. ex C. Wrigh e Prockia crucis P. Browne ex L. (Bullock 1985), C. arborea (Rich.) Urb. (Bawa et al. 1985a), P. flava (Zapata \& Arroyo 1978) e Ryania speciosa (Martiniano 2004) que são xenógamas obrigatórias. Segundo Endress (1994), a autoincompatibilidade é a principal estratégia que leva à polinização cruzada. Auto-incompatibilidade é amplamente distribuída entre as espécies arbóreas tropicais, que são predominantemente xenógamas obrigatórias (Bawa 1974, Gibbs \& Bianchi 1999). De acordo com Bawa (1974), a prevalência de sistemas de autoincompatibilidade e, por conseqüência, de polinização cruzada em árvores tropicais é provavelmente devida à pressão seletiva para a manutenção da variação genética.
Sucesso reprodutivo - O potencial reprodutivo e sucesso reprodutivo pré-emergente (SRPE) das espécies estão sumarizados na tabela 4. L. paludosa, C. grandiflora e C. javitensis apresentaram alto potencial reprodutivo, mas baixo sucesso reprodutivo pré-emergente (SRPE). De acordo com Bawa (1990) em muitas espécies de plantas nem todos os óvulos se tornam sementes. Diferentes fatores estão envolvidos no aborto de óvulos, tais como número de grãos de pólen depositados no estigma. Outra causa seria a competição entre embriões pelos recursos da planta-mãe (Bawa \& Webb 1984). Doungyotha \& Owens (2002), estudando a biologia reprodutiva e sucesso reprodutivo de Pterocarpus macrocarpus Kurz (Leguminosae:Papilionoideae), obtiveram dados similares com 0,006 de sucesso reprodutivo pré-emergente. Os autores ainda mencionaram que a causa do baixo sucesso reprodutivo foi a alta proporção de óvulos e flores abortados.

Tabela 4. Sucesso reprodutivo pré-emergente de Lindackeria paludosa, Casearia grandiflora e C. javitensis no campus da UFAM, Manaus, AM.

Table 4. Pre-emergent reproductive success of Lindackeria paludosa, Casearia grandiflora and C. javitensis at the UFAM campus, Manaus, AM.

\begin{tabular}{lccc}
\hline Características florais & L. paludosa & C. grandiflora & C. javitensis \\
\hline Número de botões & 608 & 465 & 432 \\
Número de flores abertas & 542 & 178 & 157 \\
Porcentagem de sobrevivência (\%) & 89,1 & 38,2 & 36,3 \\
Número de frutos formado por flores & 38 & 162 & 9 \\
Número de sementes formado por flores & 76 & 652 & 8 \\
Razão de frutos por flores & 0,07 & 0,91 & 0,05 \\
Razão de sementes por óvulo & 0,001 & 0,08 & 0,002 \\
Sucesso reprodutivo pré-emergente & 0,0007 & 0,07 & 0,0001 \\
\hline
\end{tabular}

Agradecimentos - Ao CNPq, pelo financiamento ao projeto "Biologia reprodutiva de espécies do sub-bosque", Processo 471064/2003-3 e aos Professores Erich Fischer e Isabel C.S. Machado, pelas correções e sugestões na dissertação.

\section{Referências bibliográficas}

BAKER, H.G. \& BAKER, I. 1983. A brief historical review of the chemistry of floral nectar. In The biology of nectaries (B. Bentley \& T. Elias, eds.). Columbia University Press, New York, p.126-152.

BAWA, K.S. 1974. Breeding systems of tree species of a lowland tropical community. Evolution 28:85-92.
BAWA, K.S. 1990. Plant-pollinator interactions, sexual systems and pollen flow - commentary.In Reproductive ecology of tropical forests plants (K.S. Bawa, \& M. Hadley, eds.). Unesco Paris, p.65-67.

BAWA, K.S. \& WEBB, C.J. 1984. Flower, fruit and seed abortion in tropical tree: implications for evolution of paternal and maternal reproduction patterns. American Journal of Botany 71:736-751.

BAWA, K.S., PERRY, D.R. \& BEACH, J.M. 1985a. Reproductive biology of tropical lowland rain forest trees. I. Sexual systems and incompatibility mechanisms. American Journal of Botany 72:331345 . 
BAWA, K.S., BULLOCK, S.H., PERRY, D.R., COVILLE, R.E. \& GRAYUM, M.H. 1985b. Reproductive biology of tropical lowland rain forest trees. II. Pollination systems. American Journal of Botany 72:346-356.

BAWA, K.S., ASHTON, P.S. \& NOR, S.M. 1990. Reprodutive ecologyof tropical forest plants: management issues. In Reprodutive ecologyof tropical forest plants (K.S. Bawa, \& M. Hadley, eds.). Unesco Paris, p.3-13.

BORBA, E.L., FELIX, J.M., SOLFERINI, V.N. \& SEMIR, J. 2001. Fly-pollinated Pleurothallis (Orquidadeae) species have high genetic variability: evidence from isozyme markers. American Journal of Botany 88:419-428.

BULLOCK, S.H. 1985. Breeding systems in the flora of a tropical deciduous forest in Mexico. Biotropica 17: 287-301.

CORBET, S.A., UNWIN, D.M. \& PRYS-JONES, O.E. 1979. Humidity, nectar and insects visits to flowers, with special reference to Crataegus, Tilia and Echium. Ecological Entomology 4:9-22.

DAFNI, A. 1992. Pollination ecology: a practical approach. Oxford University Press, New York.

DELAMONICA, P., LAURENCE, W.F. \& LAURANCE, S.G. 2001. A fragmentação da paisagem. In Florestas do Rio Negro (A.A. Oliveira \& D.C. Daly, eds.). Schwarcz, São Paulo, p.285-301.

DOUNGYOTHA, Y. \& OWENS, J.N. 2002. The reproductive biology and reproductive success of Pterocarpus macrocarpus Kurz. Biotropica 34:58-67.

DULMEN, A. 2001. Pollination and phenology of flowers in the canopy of two contrasting rain forest types in Amazonia Colombia. Plant Ecology 153:73-85.

ENDRESS, P.K. 1994. Diversity and evolutionary biology of tropical flowers. Cambridge University Press, New York.

FAEGRI, K. \& VAN DER PIJL, L. 1979. The principles of pollination ecology. Pergamon Press. Inc., New York.

GIBBS, P.E. \& BIANCHI, M.B. 1999. Does late-acting self-incompatibility (LSI) show family clustering? Two more species of Bignoniaceae with LSI: Dolichandra cynanchoides and Tabebuia nodosa. Annals of Botany 84:449-457.

HAMRICK, J.L. \& NASON, J.D. 2000. Gene flow in forest trees. In Forest conservation genetics: principles and practice (A. Young, D. Boshier \& T. Boyle, eds.). CSIRO Publishing, Melbourne, p.352-365.

JAIMES, I. \& RAMIREZ, N. 1999. Breeding systems in a secondary deciduous forest in Venezuela: the importance of life form, habitat and pollination specificity. Plant Systematics and Evolution 215:23-36.

KATO, M. 1996. Plant-pollinator interactions in the understory of lowland mixed dipterocarp forest in Sarawak. American Journal of Botany 83:732-743.

KEARNS, C.A. \& INOUYE, D.W. 1993. Techniques for pollination biologists. University Press of Colorado, Niwot.
MACHADO, A.O. \& OLIVEIRA, P.E. 2000. Biologia floral e reprodutiva de Casearia grandiflora Camb. (Flacourtiaceae). Revista Brasileira de Botânica 23: 283-290.

MACHADO, I.C.S. \& LOIOLA, M.I. 2000. Fly pollination and pollinator sharing in two synchronopatric species: Cordia multispicata (Boraginaceae) and Borreria alata (Rubiaceae). Revista Brasileira de Botânica 25: 35-42.

MARTINIANO, T.M. 2004. Biologia floral e recursos oferecidos por espécies de sub-bosque em um fragmento florestal urbano no Município de Manaus, AM. Dissertação de mestrado, Instituto Nacional de Pesquisas da Amazônia, Manaus.

MEDRANO, M., GUITIÁN, P. \& GUITIÁN, J. 1999. Breeding system and temporal variation in fecundity of Pancratium maritimum L. (Amarylliaceae). Flora 194:13-19.

MOMOSE, K., YUMOTO, T., NAGAMITSU, T., KATO, M., NAGAMASU, H., SAKAI, S., HARRISON, R.D., ITIOKA, T., HAMID, A.A. \& INOUYE, T. 1998. Pollination biology in a lowland dipterocarp forest in Sarawak, Malasia. I. Characteristics of the plantpollinator community in a lowland dipterocarp forest. American Journal of Botany 85:1477-1501.

PIRES-O'BRIEN, M.J. \& O'BRIEN, C.M. 1995. Ecologia e modelamento de florestas tropicais. Faculdade de Ciências da Administração de Pernambuco - FCAP, Serviço de Documentação e Informação, Belém.

POMBAL, E.C. 1994. Biologia floral de duas espécies dicogâmicas, Metrodorea nigra e Metrodorea stipularis (Rutaceae), em matas semidecíduas no sudeste do Brasil. Dissertação de mestrado, Universidade Estadual Paulista, Rio Claro.

POMBAL, E.C. \& MORELLATO, P.C. 1995. Polinização por moscas em Dendropanax cuneatus (DC.) Decne. \& Planch. (Araliaceae) em floresta semidecídua no sudeste do Brasil. Revista Brasileira de Botânica 18:157-162.

PRANCE, G.T. 1985. The pollination of amazonian plants. In Key environments Amazonia (G.T. Prance \& T.E. Lovejoy, eds.). Oxford Press, New York, p.166-191.

SALATI, E., SANTOS, A.A., LOVEJOY, T.E. \& KLABIN, I. 1998. Por que salvar a floresta Amazônica. Instituto Nacional de Pesquisas da Amazônia, Manaus.

SANTOS, M.J.L. \& MACHADO, I.C. 1998. Biologia floral e heterostilia em Vismia guianensis (Aubl.) Choisy (Clusiaceae). Acta Botanica Brasilica 12:451-464.

SIGRIST, M.R. \& SAZIMA, M. 2002. Ruellia brevifolia (Pohl) Ezcurra (Acanthaceae): fenologia da floração, biologia da polinização e reprodução. Revista Brasileira de Botânica 25:35-42.

SILVA, M.S., FONTENELLE, J.C.R. \& MARTINS, R.P. 2001. Por que moscas visitam flores? Ciência Hoje 30:68-71. 
SILVA-FORSBERG, M.C. 1999. Protecting an urban forest reserve in the Amazon. A multi-scale analysis of edge effects population pressure, and institutions. Dissertation Series, n.3. Center for the Study of Institutions, Population and Enviromental, Indiana University, Indiana.

SLEUMER, H.O. 1980. Flacourtiaceae. Flora Neotropica. Monograph 22. The New York Botanical Garden, New York.
THORP, R.W. 2000. The collection of pollen by bees. Plant Systematic and Evolution 222:211-223.

WIENS, D., CALVIN, C.L., WILSON, C.A., DAVERN, C.I., FRANK, D. \& SEAVEY, S.R. 1987. Reproductive success, spontaneous embryo abortion, and genetic load in flowering plants. Oecologia 71:501-509.

ZAPATA, T.R. \& ARROYO, M.T.K. 1978. Plant reproductive ecology of a secondary deciduous tropical forest in Venezuela. Biotropica 10:221-23. 